\title{
The accumulation of bactericidal lipids in staphylococcal abscesses
}

\author{
T. R. SHRYOCK*, E. S. DYE† and F. A. KAPRAL
}

Department of Medical Microbiology and Immunology, The Ohio State University, Columbus, OH 43210, USA

\begin{abstract}
Summary. Abscesses were generated in the peritoneal cavity of mice by the inoculation of $10^{9}$ staphylococci. Abscess weight increased rapidly, reaching about $200 \mathrm{mg}$ by the fourth day; for the next 60 days, abscess weight increased only slightly. The amount of total lipid increased during abscess development, attaining a peak level of about $19 \mathrm{mg}$ per abscess at 7 days before decreasing. Almost all of this lipid resulted from the accumulation of neutral lipids. The small increases seen in the phospholipid and glycolipid fractions could be accounted for through the accumulation of host cellular elements in the abscess. Leucocytes containing cytoplasmic lipid droplets were first seen 4-12 $\mathrm{h}$ after infection and these cells were widely scattered around the periphery. During the next 2 days, the number of cells with lipid droplets increased markedly and lipid droplets were also found in the deeper portions of the abscesses. Although lipid droplets were found subsequently throughout the abscess, the greatest amounts always occurred in the leucocyte zone immediately proximal to the connective tissue capsule. During abscess development, the bactericidal activity also increased rapidly, reaching a maximum by the seventh day and declining thereafter.
\end{abstract}

\section{Introduction}

When Staphylococcus aureus strains possessing clumping factor are inoculated into the peritoneal cavity of mice, they are promptly clumped through the interaction of the clumping factor and fibrinogen in the peritoneal fluid. ${ }^{1}$ During the ensuing inflammatory response, the clumped organisms become surrounded by a thick layer of leucocytes, but phagocytosis is negligible because leucocytes in proximity to the cocci are quickly disrupted. Over the next 4 days, the clumps coalesce and become enclosed by connective tissue. At this stage, the abscesses consist of a core of densely packed cocci, a broad zone of acellular debris, a zone of disintegrating leucocytes, a region of intact leucocytes and a vascularised connective-tissue capsule. ${ }^{2}$

Staphylococci exhibit three distinct patterns of survival within abscesses. Some strains are destroyed rapidly whereas others persist for 2-3 months. Still other strains are eliminated, but only after a delay of 710 days. $^{2.3}$ The destruction of staphylococci does not result from phagocytosis or from the release of lysosomal substances, but rather from the production of bactericidal lipids which affect strains to a different extent depending on their survival pattern. ${ }^{4.5}$

Received 21 March 1991; accepted 10 July 1991.

* Present address: Indiana State University, Terra Haute, IN 47809, USA

† Present address: Trudeau Institute. Saranac Lake, NY 12893 , USA.
Although abscess homogenates exhibit a certain amount of bactericidal activity due to these lipids, the amount of activity can be increased by first incubating homogenates with either live staphylococci or their sterile culture filtrates. This phenomenon has been termed "activation"., ${ }^{3,4}$ The basis for activation is not understood, but the process may represent conversion of an inactive precursor to bactericidal lipids.

Because of the involvement of lipids in this type of host-parasite interaction, efforts were made to measure the amount of lipid present during the course of abscess maturation and also to determine its location histologically.

\section{Materials and methods}

\section{Staphylococcal strains}

S. aureus strains $303,18 \mathrm{Z}$ and TG have been described previously. ${ }^{3}$ These strains are representative of organisms that exhibit the immediate elimination, delayed elimination, or long-term persistence patterns of survival, respectively. Abscesses in which lipids and bactericidal activity were measured were generated with $S$. aureus strain 18Z; abscesses for histological studies were produced with strains $18 \mathrm{Z}, 303$ or TG. Strain 303 also served as the indicator organism in assays of abscess homogenates for bactericidal activity. Suspensions of staphylococci were washed, counted and stored frozen until needed. 


\section{Generation of intraperitoneal abscesses}

Abscesses were produced as described previously. ${ }^{3}$ Groups of white Swiss mice (Harlan/Sprague-Dawley Inc. Indianapolis, IN, USA), were inoculated intraperitoneally with $10^{9}$ cocci in $0.25 \mathrm{ml}$ of diluent. Beginning $4 \mathrm{~h}$ after inoculation and at various intervals thereafter over the next 70 days, animals were killed and either the leucocyte-covered clumps or abscesses were collected. All abscesses obtained from a single animal were pooled and weighed.

\section{Preparation of abscess homogenates}

At each sample time, the abscesses from four to eight mice were pooled, homogenised in saline ( $1 \mathrm{ml} /$ animal), and heated to $100^{\circ} \mathrm{C}$ for $30 \mathrm{~min}$ to kill residual staphylococci. ${ }^{3}$ Separate homogenates were prepared for lipid analyses and for the measurement of bactericidal activity. For activation, samples of homogenates were mixed with three volumes of a sterile culture filtrate prepared from an overnight culture of $S$. aureus $18 \mathrm{Z}$ in trypticase soy broth. The mixtures were incubated at $37^{\circ} \mathrm{C}$ for $24 \mathrm{~h}$ and assayed for bactericidal activity. The end points were subsequently corrected for the dilution necessitated by the addition of culture filtrate.

\section{Lipid analyses}

Homogenates prepared from pools of abscesses collected from four to eight mice were extracted according to the Bligh and Dyer modification of the Folch procedure. ${ }^{6}$ Non-lipid contaminants were removed by partition chromatography on Sephadex G- $25^{7}$ and the lipid extract was separated into neutral lipid, glycolipid and phospholipid fractions by chromatography on silicic acid (Unisil) columns. ${ }^{5,8}$

\section{Histological preparations}

Individual abscesses collected at various times after infection were fixed in neutral buffered formalin and frozen sections were cut from different parts of the specimen. At each location, one section was stained with Oil Red $\mathrm{O}$ to reveal lipid ${ }^{9}$ and an adjacent section was stained with Haematoxylin-Eosin to confirm the various regions within the abscess. ${ }^{2}$

\section{Assay of bactericidal activity}

The procedure has been described in detail. ${ }^{3}$ Serial two-fold dilutions of homogenate were made in $2 \mathrm{M}$ $\mathrm{NaCl}$ containing $2 \mathrm{mM}$ EDTA and an appropriate amount of $S$. aureus 303 suspension was added. After incubation of $37^{\circ} \mathrm{C}$ for $1 \mathrm{~h}$ the surviving cocci were counted as cfu. A cidal unit (ED50) was determined by the method of probits and bactericidal activity was expressed as ED50/mouse. Each homogenate was assayed in duplicate and the results were averaged.

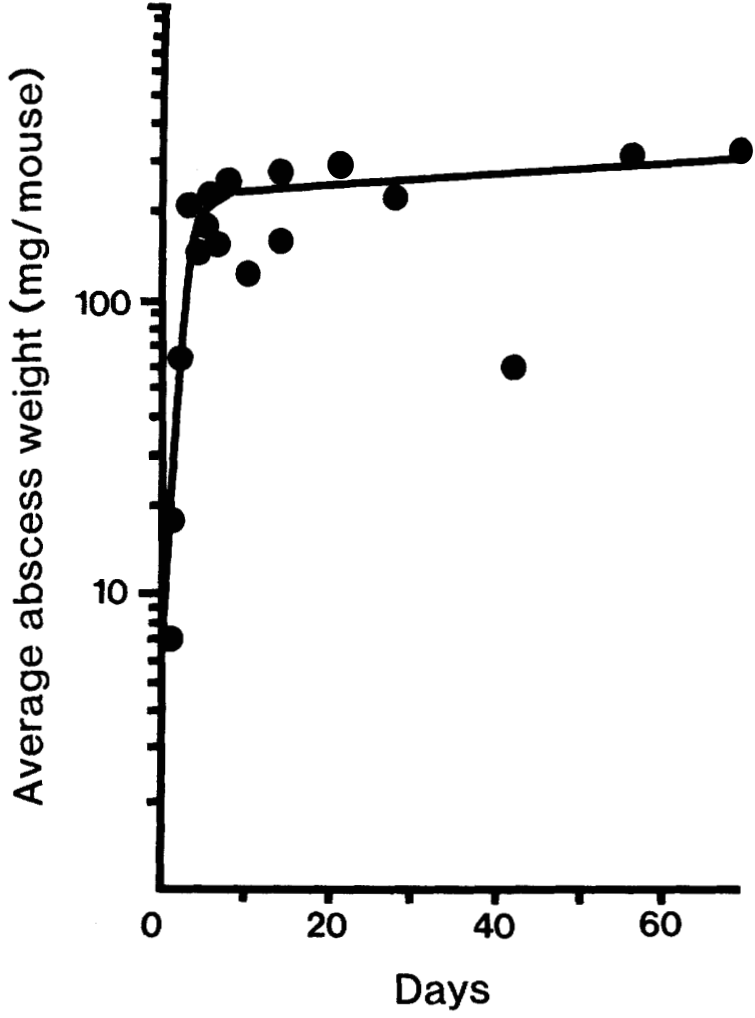

Fig. 1. Average weight of abscesses generated in the peritoneal cavity of mice inoculated with $10^{9} \mathrm{cfu}$ of $S$. aureus 18Z. Each point represents the average weight of abscesses from four to eight mice.

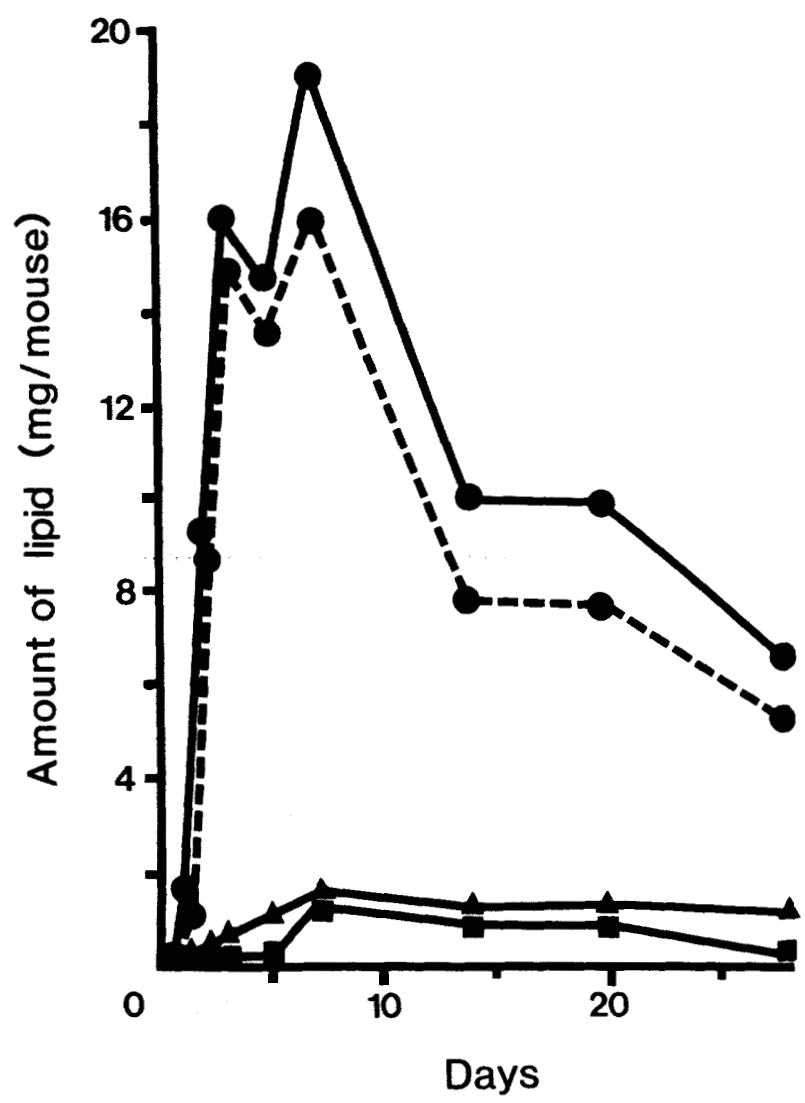

Fig. 2. The amount of lipids ( - , total) present in abscesses generated by the intraperitoneal inoculation of $10^{9} \mathrm{cfu} S$. aureus $18 \mathrm{Z}$. Abscesses from four to eight mice were homogenised and the lipids were extracted by the Folch procedure. The lipids were separated into neutral lipid (-- glycolipid (phospholipid $(\boldsymbol{\Delta}-\mathbf{\Delta})$ fractions by chromatography on silicic acid. 


\section{Results}

\section{Abscess weight}

During the first 4 days after infection, the period over which lesions develop into mature abscesses, the mean lesion weight rapidly increased about 30 -fold. Thereafter, for the next several weeks, the mean abscess weight continued to increase albeit much more slowly (fig. 1).

\section{Lipid in abscesses}

Very little lipid was extracted from leucocytecovered clumps removed $12 \mathrm{~h}$ after infection, but by $24 \mathrm{~h}$ the developing abscesses from a single animal
Table. Lipid content of abscesses

\begin{tabular}{|c|c|c|c|c|}
\hline \multirow{2}{*}{ Day } & \multicolumn{4}{|c|}{ Percentage of wet weight } \\
\hline & Total lipid & Neutral lipids & Phospholipids & Glycolipids \\
\hline 0.5 & & 2.5 & & \\
\hline 1 & $8 \cdot 3$ & 7.2 & 1.0 & 0.6 \\
\hline 2 & 13.8 & 12.6 & 0.60 & 0.2 \\
\hline 3 & 10.5 & $10 \cdot 0$ & 0.40 & 0.1 \\
\hline 5 & $7 \cdot 1$ & 6.6 & 0.51 & $0 \cdot 1$ \\
\hline 7 & $8 \cdot 3$ & 7.0 & 0.70 & 0.6 \\
\hline 14 & $4 \cdot 1$ & $3 \cdot 2$ & 0.52 & 0.4 \\
\hline 20 & 3.8 & 3.0 & 0.49 & 0.4 \\
\hline 28 & $2 \cdot 3$ & 1.9 & 0.43 & 0.1 \\
\hline
\end{tabular}
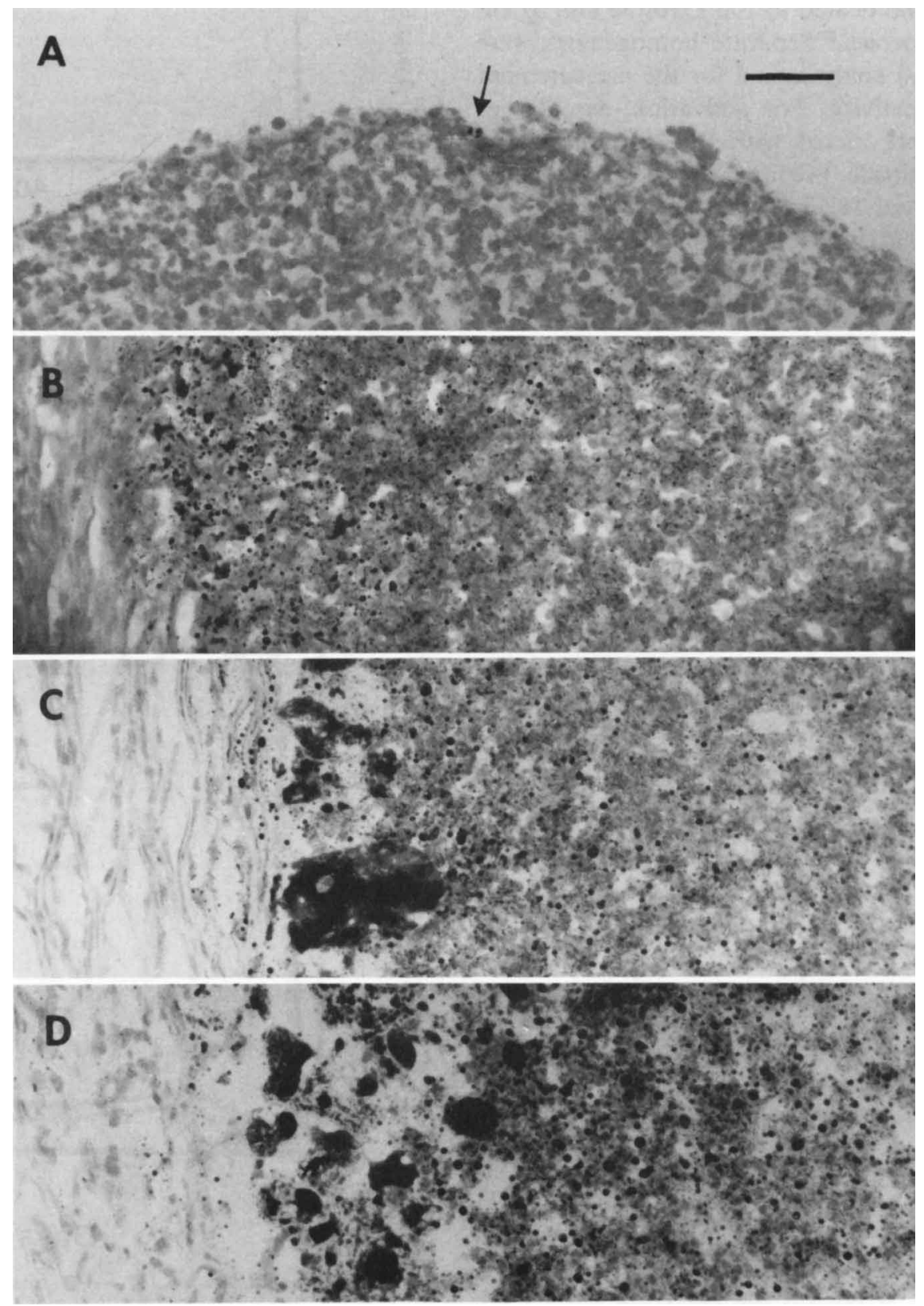

Fig. 3. Lipid accumulations in staphylococcal abscesses during various stages of development. Sections were stained with Oil Red $O$ and photographed with a green filter to enhance contrast. Lipid droplets appear black. All panels are at the same magnification. Bar $=20 \mu \mathrm{m}$. A Section through leucocyte-covered staphylococcal clump removed $4 \mathrm{~h}$ after infection. Only an occasional cell at the extreme periphery of the leucocyte layer contains lipid droplets at this time (arrow). The centre of the lesion is toward the bottom. B-D. Sections through abscesses removed 7.30 and 75 days after infection, respectively. The connective tissue capsule is on the left and the abscess core is toward the right. 
contained about $1.6 \mathrm{mg}$ total lipid (fig. 2). Peak lipid levels of about $19 \mathrm{mg} / \mathrm{mouse}$ were found at 7 days. Thereafter, the lipid content declined even though abscess weight remained relatively unchanged.

A fairly constant proportion (78-95\%) of the total lipid fraction consisted of neutral lipids. Consequently, the amount of neutral lipids paralleled the amount of total lipid present, attaining a maximum of about $16 \mathrm{mg} / \mathrm{mouse}$ by the seventh day. However, if viewed as a percentage of abscess weight, peak values occurred on day 2 , when the neutral lipids comprised $12.6 \%$ of the wet weight (table). Thereafter, the values decreased rapidly, accounting for only $3.2 \%$ of the abscess weight on day 14 and $1.9 \%$ on day 28 .

Although the amounts of phospholipids and glycolipids increased during the first 7 days, these increases probably resulted from the accumulation of cellular elements during abscess maturation since the concentration of these lipids, as percentage of wet weight, remained relatively constant for 28 days (table). During this period, phospholipids averaged $0.52 \%$ of wet weight (range $0.4-1.0 \%$ ) and glycolipids $0.2 \%$ (range $0.1-0.6 \%$ ).

\section{Histological studies}

Frozen sections stained with Oil Red O revealed that only an occasional cell contained lipid droplets during the first 4-12 h after infection. These cells appeared to be macrophages, and at this time were widely scattered around the extreme periphery of the leucocyte layer (fig. 3 A). By $24 \mathrm{~h}$, sufficient lipid-laden cells had accumulated to form an almost continuous layer around the periphery of the developing abscess.

Over the next 2 days, as the leucocyte layer became enclosed by the connective tissue capsule, numerous small lipid droplets appeared among the leucocytes located in the deeper portions of this region. After 4-7 days, droplets of lipid were present throughout the structure, even in the core of the lesion which consisted of cocci and debris, but no intact leucocytes. However, the greatest accumulation of lipid continued to be found among those leucocytes present just beneath the connective tissue capsule (fig. 3B). Although this overall pattern of lipid distribution persisted for at least a 10 -week period, with time considerable enlargement of droplets occurred near the periphery of the leucocyte region, presumably through coalescence of smaller droplets (fig. 3C). This enlargement of lipid droplets was also evident in the deeper regions of the abscess (fig. 3D). The sequence of lipid accumulation was the same regardless of the staphylococcal strain used to generate the abscesses.

\section{Bactericidal activity within abscesses}

No bactericidal activity was detected in homogenates prepared from leucocyte-covered clumps recovered $12 \mathrm{~h}$ after infection, but low levels of activity were present in the 24-h samples (fig. 4). In subsequent

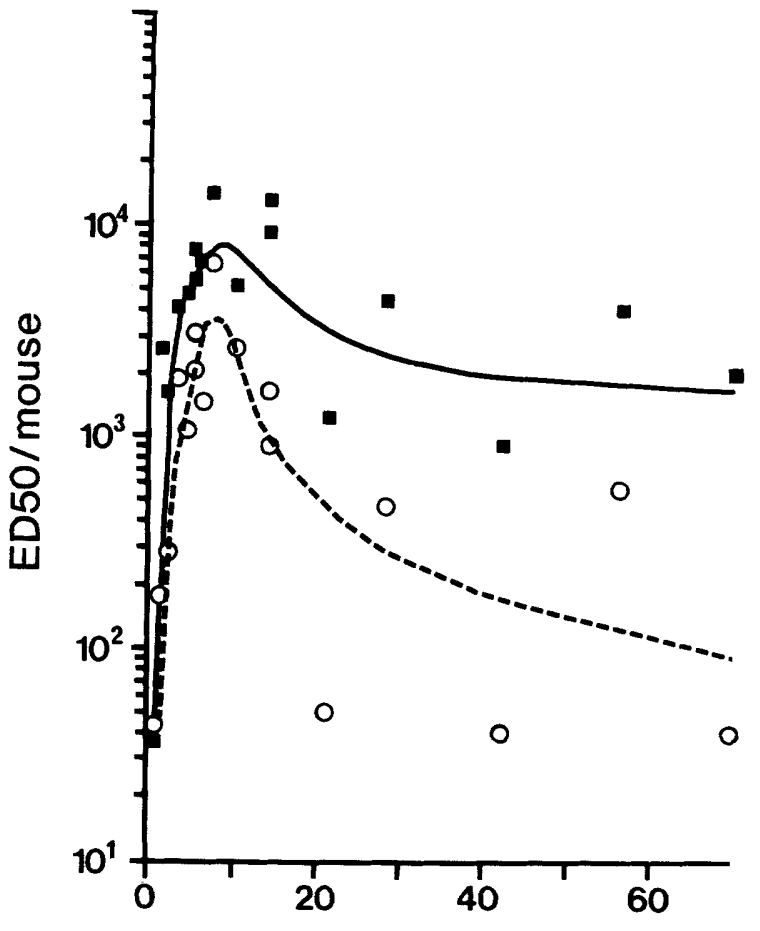

Days after inoculation

Fig. 4. Bactericidal activity in abscesses generated by the intraperitoneal inoculation of $10^{9} \mathrm{cfu}$ of $S$. aureus $18 \mathrm{Z}$. Abscess homogenates were assayed before $\left(\mathrm{O}_{--}-\mathrm{O}\right)$ and after $(\mathbf{a}-\mathbf{a})$ activation. Each point represents results obtained with homogenates prepared with abscesses from four to eight mice.

samples, the level of bactericidal activity rose sharply until day 7 when peak levels in excess of 3000 ED50/mouse were attained. During the remainder of the 10 -week observation period, bactericidal activity declined slowly.

With the exception of the 12-h samples prepared from leucocyte-covered clumps, all homogenates showed some increase in bactericidal activity after activation. The extent to which activation resulted in augmented activity increased progressively over the 70-day period of observation.

\section{Discussion}

The composition of the total lipid fraction remained fairly consistent with neutral lipids accounting for c. $90 \%$, and phospholipids and glycolipids for $9 \%$ and $1 \%$ respectively. This was the case regardless of whether the homogenate had been activated or not. It has been reported that lipids extracted from microbial or animal sources generally consist of $60-85 \%$ phospholipid and glycolipid $;^{8}$ thus, the high neutral lipid content in abscesses is distinctly unusual. However, it should be noted that injured or hypoxic tissue culture cells have been found to contain high levels of triglycerides and free fatty acids. ${ }^{10}$

It requires about 4 days for the intraperitoneal lesions to develop into mature abscesses with a vascularised connective tissue capsule. ${ }^{2}$, During this time, the average lesion weight increased from $7 \mathrm{mg}$ to 
$200 \mathrm{mg}$ (fig. 1). Since bacterial multiplication does not occur in these lesions, ${ }^{2.3}$ most of the increase in weight must have resulted from the deposition of host components such as leucocytes and connective tissue. The accumulation of cellular elements probably accounts for the small increases in phospholipid and glycolipid levels seen in abscess extracts during the first few days. These conclusions are supported by the observation that the phospholipid and glycolipid levels, when viewed as a percentage of abscess wet weight, remained relatively constant. This would be expected if they are contributed by membranes of the cells accumulating in the lesions (table). The much greater accumulation of neutral lipids appears to result through a different mechanism.

Histological sections of mature lesions, when stained with Oil Red $O$, revealed large accumulations of lipid at the periphery of the abscess in the layer of intact leucocytes, with smaller droplets scattered throughout the deeper regions consisting of acellular debris, and even into the abscess core where the viable cocci are located. While most of the stainable lipid initially consisted of small droplets found in the cytoplasm of cells that appeared to be macrophages, in later samples the accumulations were much greater in size and appeared to be extracellular.

The leucocyte layer in abscesses consists primarily of neutrophils, but some macrophages are also present. ${ }^{2}$ This layer of viable leucocytes just beneath the connective tissue capsule remains a prominent feature in abscesses even after many weeks. In view of the short life span of neutrophils, it seems probable that maintenance of this zone results from a constant influx of fresh cells from the vascularised capsule. This site is also where the greatest amounts of lipid are accumulated, suggesting that lipid production or accumulation is associated with cells which have recently entered the abscess. Lipid accumulation may depend upon the arrival of cells with an altered metabolism, perhaps brought on by substances released in the peritoneal cavity after infection. In keeping with this hypothesis are recent observations (unpublished) in mice infected intraperitoneally with low doses of $S$. aureus. We found that the original resident macrophages failed to accumulate lipid, but a population of macrophages arriving subsequently accumulated substantial amounts of lipid in their cytoplasm. These newly arriving cells are larger and spread more easily on surfaces than do the macrophages originally present.

$S$. aureus $18 \mathrm{Z}$ exhibits a pattern of delayed elimination within abscesses. ${ }^{2}$ When this strain was used to generate abscesses, maximal levels of bactericidal activity in abscess homogenates occurred at 7 days which coincides with the commencement of the elimination phase. Peak levels of bactericidal activity also coincided with maximal levels of neutral lipids. Whereas the neutral lipid fraction exhibited bactericidal activity, this was not the case for the phospholipid or glycolipid fractions. This agrees with previous findings that the bactericidal activity in staphylococcal abscesses is due to a pool of free fatty acids and another as yet unidentified lipid, both of which are found in the neutral lipid fraction. ${ }^{5}$

With the exception of homogenates prepared from leucocyte-covered clumps, all homogenates revealed appreciable increases in bactericidal activity after activation. If the phenomenon of activation does indeed involve conversion of an inactive precursor, this would suggest that little or no precursor is present in leucocyte-covered clumps but that the amount of precursor increases steadily as abscesses develop and mature.

This investigation was supported by Public Health Service Grants AI-7826 and AI-19879 from The National Institute of Allergy and Infectious Disease.

\section{References}

1. Kapral FA. Clumping of Staphylococcus aureus in the peritoneal cavity of mice. J Bacteriol 1966; 92: 1188-1195.

2. Kapral FA, Godwin JR, Dye ES. Formation of intraperitoneal abscesses by Staphylococcus aureus. Infect Immun 1980; 30 $204-211$.

3. Dye ES, Kapral FA. Survival of Staphylococcus aureus in intraperitoneal abscesses. J Med Microbiol 1981; 14 $185-194$.

4. Dye ES, Kapral FA. Partial characterization of a bactericidal system in staphylococcal abscesses. Infect Immun 1980; 30 198-203.

5. Dye ES, Kapral FA. Characterization of a bactericidal lipid developing within staphylococcal abscesses. Infect Immun 1981: 32: $98-104$
6. Bligh EG, Dyer WJ. A rapid method of total lipid extraction and purification. Can J Biochem Physiol 1959;37:911-917.

7. Rouser G, Kritchevsky G, Yamamota A. Column chromatographic and associated procedures for separation and determination of phosphatides and glycolipids. In: Marinetti GV (ed) Lipid chromatographic analysis, vol 1. New York, Marcel Dekker, Inc. 1967: 99-162.

8. Kates M. Techniques of lipidology: isolation, analysis and identification of lipids. Amsterdam, North Holland Publishing Company. 1972.

9. Culling CF. Handbook of histopathological techniques, 2nd edn. London, Butterworths. 1963: 289-306.

10. Gordon GB, Barcza, MA, Bush ME. Lipid accumulation in hypoxic tissue culture cells. Am J Pathol 1977; 88: 663-678. 